\title{
Brain Imaging Procedures and Surgery Techniques: Past, Present and Future
}

\author{
Shweta Suran ${ }^{1}$, Vishwajeet Pattanaik ${ }^{1}$, Mayank Singh ${ }^{1}$, P.K. Gupta ${ }^{2}$, and Praveen \\ Gupta $^{3}$ \\ ${ }^{1}$ Computer Science \& Engineering Department, Krishna Engineering College, \\ Ghaziabad, India \\ \{shwetasuran10, vpattanaik, mayanksingh2005\}@gmail.com \\ ${ }^{2}$ Department of Computer Science \& Engineering, Jaypee University of \\ Information Technology, Solan, India. \\ pkgupta@ieee.org \\ ${ }^{3}$ Neurology Department, Fortis Memorial Research Institute, Gurgaon, India. \\ praveenguptapg@gmail.com
}

\begin{abstract}
Desk bound jobs, inactive lifestyles and changing habits have made the occurrence of strokes and tumors more extensive among South Asians, especially Indians and can generate permanent disability or prove fatal. Diagnostic Imaging plays a vital role in diagnosis of brain tumors and strokes. Diagnostic Medical Imaging delivers essential information about our health to physicians, neurologists, emergency room neurosurgeons and other physicians. The scientific knowledge in medical imaging has revolutionized management of health care over the past 3 decades, it has enabled doctors to find symptoms of diseases earlier and hence improve the patient's health faster. In case of tumors and strokes, latest medical imaging techniques have improved on both diagnosis and intervention. Tumors and strokes are treated with surgery, mainly Minimal Invasive Neurosurgery with state-of-art imaging technologies offering highly personalized and direct means of cutting out brain tumors and strokes while leaving healthy tissues of the brain untouched. In near future, Image Guided - Minimal Invasive Neurosurgery would allow for little cerebrospinal fluid loss, no brain retraction, less blood loss and reduction in seizures. The upcoming generation of Minimal Invasive Surgery will increase the survival rate of patients and protect patients from the trauma during neurosurgeries.
\end{abstract}

Keywords: Cyber Knife Surgery, Gamma Knife Surgery, Computed Tomography (CT), Functional Magnetic Resonance Imaging (fMRI), Magnetic Resonance Angiography (MRA), Magnetic Resonance Imaging ( MRI), Medical Imaging, , Minimal Invasive Surgery

\section{Introduction}

In the past years, diseases have been the root cause of deaths in India. Coronary Artery Diseases, Strokes, Cancers, Tumors, Diabetes Mellitus, Tuberculosis (TB) are major fatal diseases in the nation. Out of these brain strokes and tumors are on the top of the list after heart diseases. Statistics provided by the National Cancer Registry Program of the India Council of Medical Research (ICMR) has stated that the biggest cause of deaths in India is Cancer; primarily Blood Cancer, Brain Cancer, Breast Cancer, Cervical Cancer, Lung Cancer and Prostate Cancer. The condition is so grave that each day around 1300 souls are lost, closing up-to approximately 500,000 deaths every year. 
Countless Indian citizens are being faced with catastrophic diagnosis each year. Brain tumours cause the deaths of more children and adults (under the age of 40) than any other cancer [19]. According to National Brain Cancer Institute in the U.S. of all primary Central Nervous System (CNS) Tumours / Primary Cerebral Lymphoma about 85 \% - 90 $\%$ cases are of Brain Tumors. There are close to 300,000 cases of tumors worldwide, out of which $10 \%$ are from India. In India about 2 patients per 100,000 people suffer from brain tumor. The morality rate (deaths due to brain cancer) in India is little less than 2 patients per 100,000 people. The Brain Tumour Foundation of India has stated that, each year approximately 40,000 - 50,000 people are identified with brain tumour; out which $20 \%$ are children. Each year more than 2,500 Indian children suffer from Medulloblastoma, which is a common type of paediatric malignant primary brain tumour originating in the cerebellum or posterior fossa.

According to the World Health Organisation (WHO) in the year 1990 there were 9.4 million deaths in India, out of which 619,000 deaths were due to stroke; giving it a mortality rate of 73 per 100,000 populations. Compared to developed countries stroke associated mortality rate among Indians has been found to be two to three times higher. Stroke burden in India is rising day by day [20]. In 2015, India accounted 1.6 million cases of stroke annually, at least one-third these were disabled due to the same. According to WHO estimates by 2050, low and middle income countries mainly India and China would account for around 80\% stroke cases from around the world.

\section{Brain Tumor}

Intracranial neoplasm most known as brain tumor is the unusual growth of cells that have reproduce in an uncontrolled fashion [15]. The brain is protected by a very rigid structure known as the human skull; any growth inside such a restricted space can cause complications. According to Brain Tumor Research Organization in U.K., there are more than 120 types of Brain Tumors each of which are graded according to how aggressive they are. Lower-grade tumors are known as Benign (Non-Cancerous) and higher-grade tumors known as Malignant (Cancerous) [16].

It is tough to predict how much time it will take for an individual with a brain tumor to show symptoms because not all brain tumors are associated with the same symptoms, and some tumors are not even uncovered until after death. Some malignant tumors, often only become visible once they have begun to spread. Almost half of malignant tumor patients are diagnosed too late, and once a cancer in the tumor has spread, it is often harder to treat it successfully.

Most common tumor types found in adults include Gliomas (Astrocytoma) and Meningiomas, whereas Astrocytomas, Medulloblastomas, Ependymomas and Brain Stem Gliomas are more commonly found in children [17]. The Prime cause of cancer related death in patients under the age of 40 is Brain Tumor. The chances of Brain Tumor in adults, is generally highest between the ages of 40 to 60; whereas in children the incidence is highest before the age of 1 and between ages of 2 and 12 . 


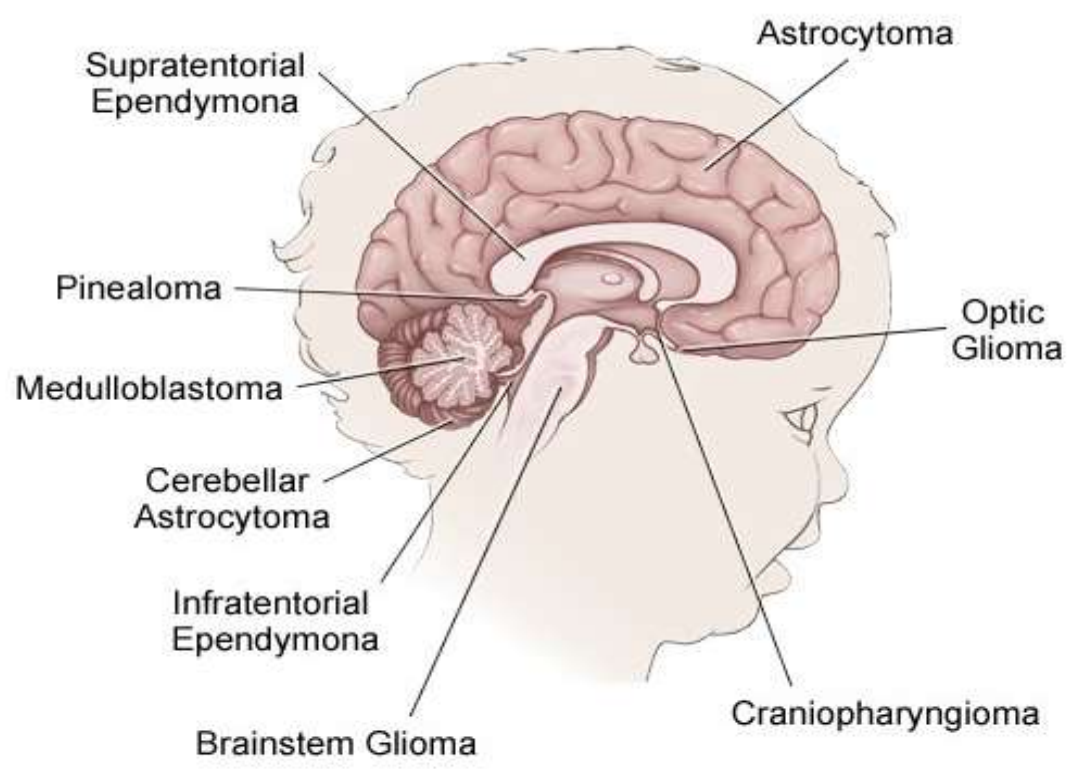

\section{Figure 1. Location of Different Types of Tumors in the Brain}

\subsection{Causes of Brain Tumor}

Brain tumor may develop at any point of time in the life and the cause of it is still unknown; although there are some genetic and environmental factors which may contribute to the development of a tumor in the brain. As a person's age increases the chances of occurrence of brain tumor also increases. The age factor nature depends upon the cell type and location of the tumor in the brain. Adults have a very low risk of developing medulloblastomas, while the risk of developing gliomas is usually higher. The occurrence of meningiomas and craniopharyngiomas is more probable in adults over the age of 50, but again these tumors may develop at any age. Chances of lymphomas occurring in the brain are higher in those people who have low/compromised immune body systems. Family history and industry chemical exposure also increases the chances of developing brain tumor. Exposure to radiation therapy or any radiation treatment at the early stage of life i.e. at a young age may increase the chances of developing tumor.

\subsection{Clinical Symptoms \& Signs of Brain Tumor}

Clinical symptom of brain tumor depends upon the type and location of the tumor; and how it interrupts that part of the brain from working properly. Severe headache during early morning hours is the most common symptom of tumor in the brain. Sudden involuntary movements of a person's muscles known as convulsions and change in sensation, vision, smell, and/or hearing without losing consciousness are other major clinical symptoms of tumor. Some symptoms like Tonic - Clonic (Grand Mal) \& Myclonic caused by other conditions like epilepsy may also lead to brain tumor.

\section{Stroke}

A cerebrovascular accident or a brain attack is known as stroke [1]. A stroke, damages the brain by interrupting adequate blood supply. It is a life threatening situation in which every seconds counts, it cannot be cured right away, but quick treatment may help in saving the patient's life. It can happen to anyone at any point of time in the life [13]. If the supply of blood to the brain isn't restated, permanent damage may occur. Damage caused 
by stroke depends on which part of the brain has suffered harm, to what degree and how fast the blood supply is restored to the part of the brain [14]. There are different types of strokes like: ischaemic stroke (caused by blocked arteries), haemorrhagic stroke (caused due to bleeding in the brain) and transient ischaemic attack or, TIA (also referred to as mini stroke) [1].

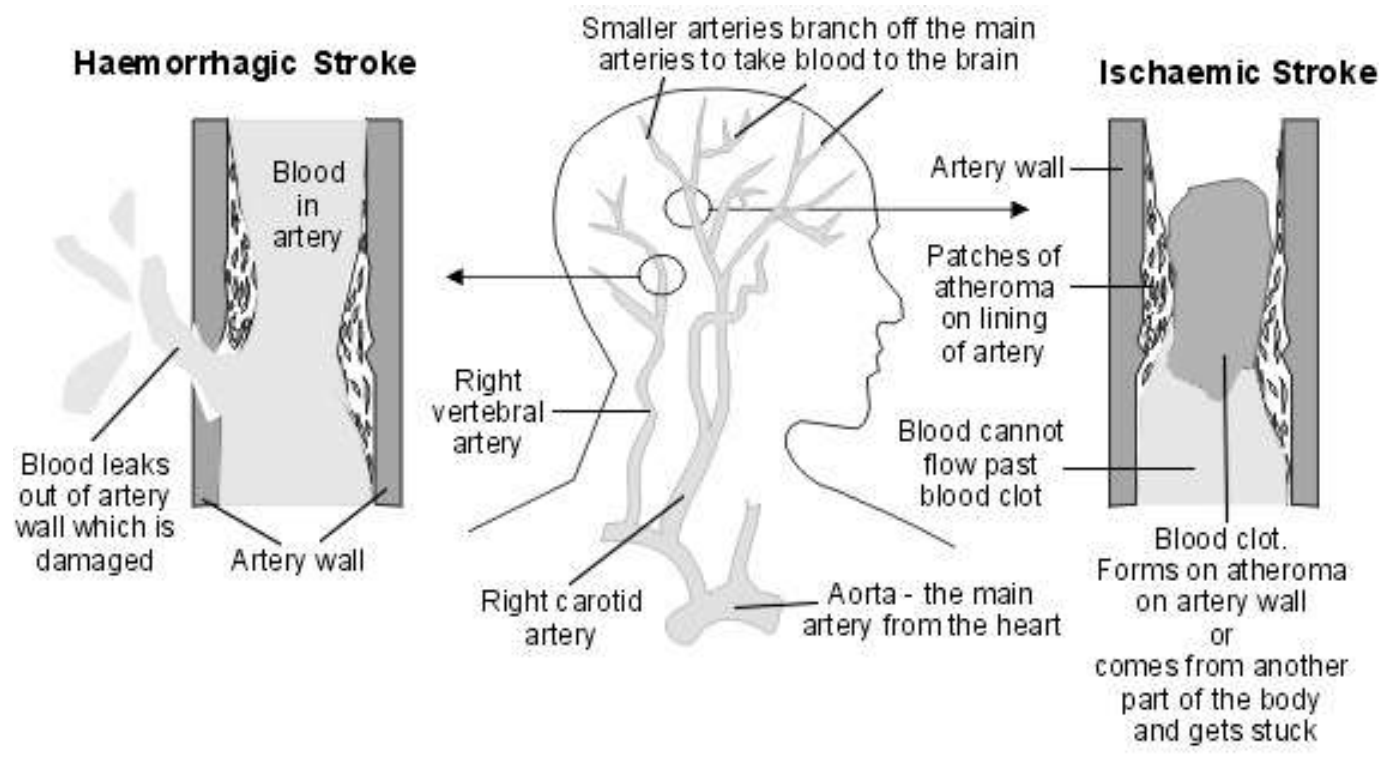

Figure 2. Different Types of Strokes

Every year 20 million people suffer from stroke worldwide, of which 5 million die and another 5 million are physically disabled. It is estimated that one out of six people suffer a stroke in their lifetime. In India, 1.5 million suffer from stroke every year and 3,000 to 4,000 are affected by it each day. What is even more distressing is that $40 \%$ die after a major stroke, $30 \%$ need full medical support and more than $50 \%$ aren't able to go work after a stroke attach.

\section{Causes of Stroke}

Several diseases responsible for stroke include high blood pressure, diabetes mellitus, arrhythmia, high cholesterol and plaques. Plaque is the build-up of cholesterol containing fatty deposits or, arteriosclerosis. These deposits occur in the blood vessel walls and can narrow blood vessels that provide blood supply to the brain. Other common medical conditions that may cause stroke include angina and peripheral vascular disease (or, poor arterial circulation to the legs).

An easier way to remember the sudden signs of stroke is F.A.S.T. [18]. F.A.S.T. is:

- F: Face Drooping

- A: Arm Weakness

- S: Speech Difficulty

- T: Time to Call

If someone shows any of these symptoms or even if the symptoms go away, one should call for the ambulance right away and get the person to the hospital immediately.

\section{Medical Imaging/Diagnostic Imaging}

Diagnostic Imaging or Medical imaging is a significant part in the early detection, analysis and cure of tumor and stroke. Fast development in the field of computer science gives a new direction in the medical imaging, it encloses distinct imaging modalities and 
processes to image the body of a person for detail dissection and cure determination and thus plays a critical role to improve health of all patients. Previously X-ray was the only method to detect brain tumor and stroke but with the advent of modern computers, new tomographic imaging modalities as Computed Tomography (CT), Magnetic Resonance Imaging (MRI), Functional magnetic resonance imaging (fMRI), MRI Angiography (MRA), Magnetic Resonance Spectroscopy (MRS), enable doctors to diagnose a brain tumor and stroke with greater precision than ever before. The more precise the diagnosis, the better the treatment. Medical imaging processing give a detailed structure and location of the problem. With the help of these imaging technologies, surgeons can rule out minimal invasive surgery with more precision [11].

\subsection{Magnetic Resonance Imaging (MRI)/Nuclear Magnetic Resonance Imaging}

It is very essential imaging approach used diagnose tumors in the soft tissue of the brain. Magnetic resonance imaging (MRI) uses a powerful magnetic field, which gives detail information of exquisite soft tissue, and anatomic of the brain [10]. Unlike CT, MRI scans do not require any ionization radiation. MRI is a special technique specifically useful in diagnosing brain tumors because it can "see" through the bones of the skull to the tissue underneath.

In soft tissue tumor imaging the prime aim is that imaging should be able to reach a definitive diagnosis or to precise the differential diagnosis and to advice whether biopsy, surgical intervention, or simple examination is required for further processing. In extension to contributing towards analysis and diagnosis, medical imaging has significant role in the staging of soft tissue malignancies and likely in response judgement. Medical imaging can precisely depict the morphology of lesions including size, location, and extent $[12,13]$. Able to provide advantageous report related to the underlying biology of lesions, often capable to illustrate the underlying physical composition of tumors and adequately limit the differential diagnosis for skeletal lesions.

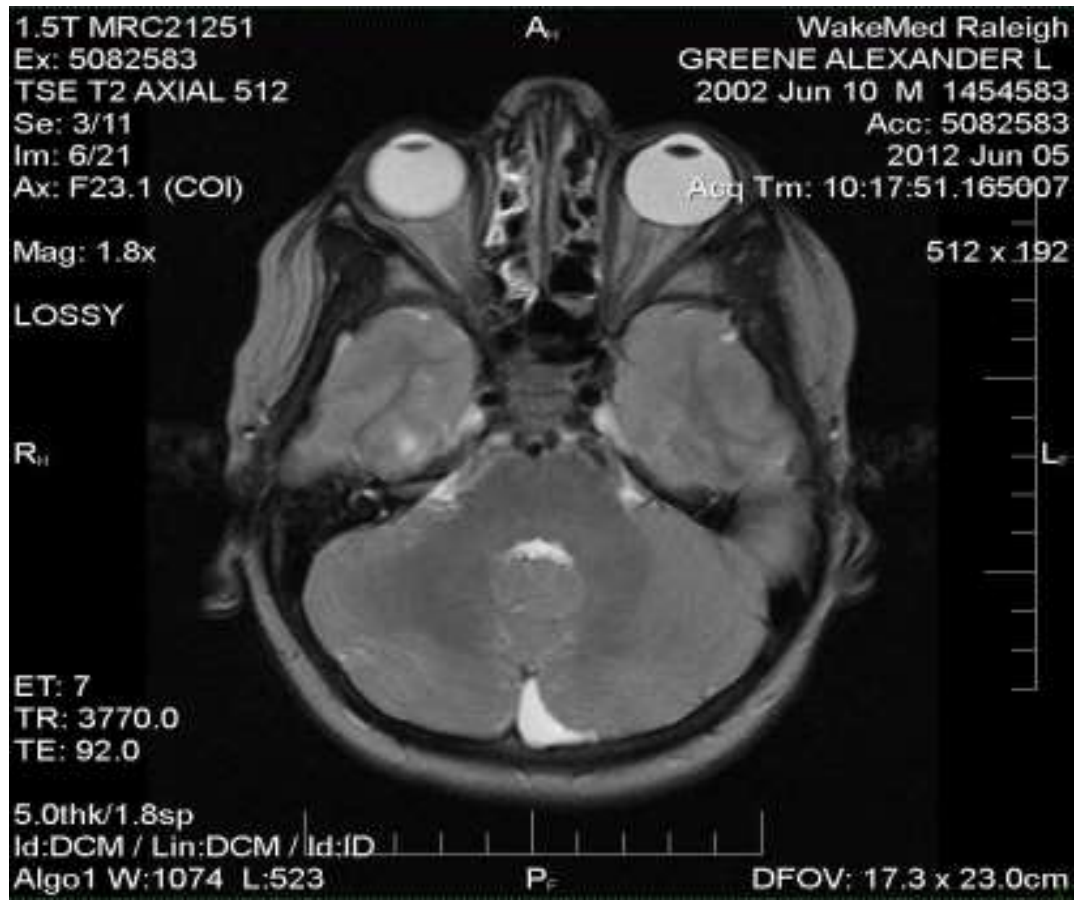

Figure 3. MRI of Subependymal Giant Cell Astrocytoma (SEGA) is a LowGrade Astrocytic Brain Tumor (astrocytoma) that Arises within the Ventricles of the Brain 


\subsection{Functional Magnetic Resonance Imaging (fMRI)}

In recent advancement as imaging modalities fMRI is a relatively modern procedure that uses MR imaging to measure the miniature metabolic changes that take place in an alive part of the brain [17]. Functional MRI is used for the determination of neurosurgical planning and neurologic threat assessment in the surgery of brain tumors and other brain related problems [21]. It localizes the eloquent cortices controlling language, motor, and memory functions. The outcome of an fMRI study can change a neurosurgical approach to a tumor, suggest that surgery is a risk-free option in cases in which it might not otherwise have been offered, or steer a clinician away from neurosurgery and towards further treatment alternatives when the risk is high.

This test is used to observe the growth and function of tumors as well as check out the areas in the brain related to language and muscle movement. The central forms of fMRI involve blood-oxygen-level dependent (BOLD) contrast. BOLD signals are universally used as a proxy for brain movement, because neurons use high oxygen when they are active.

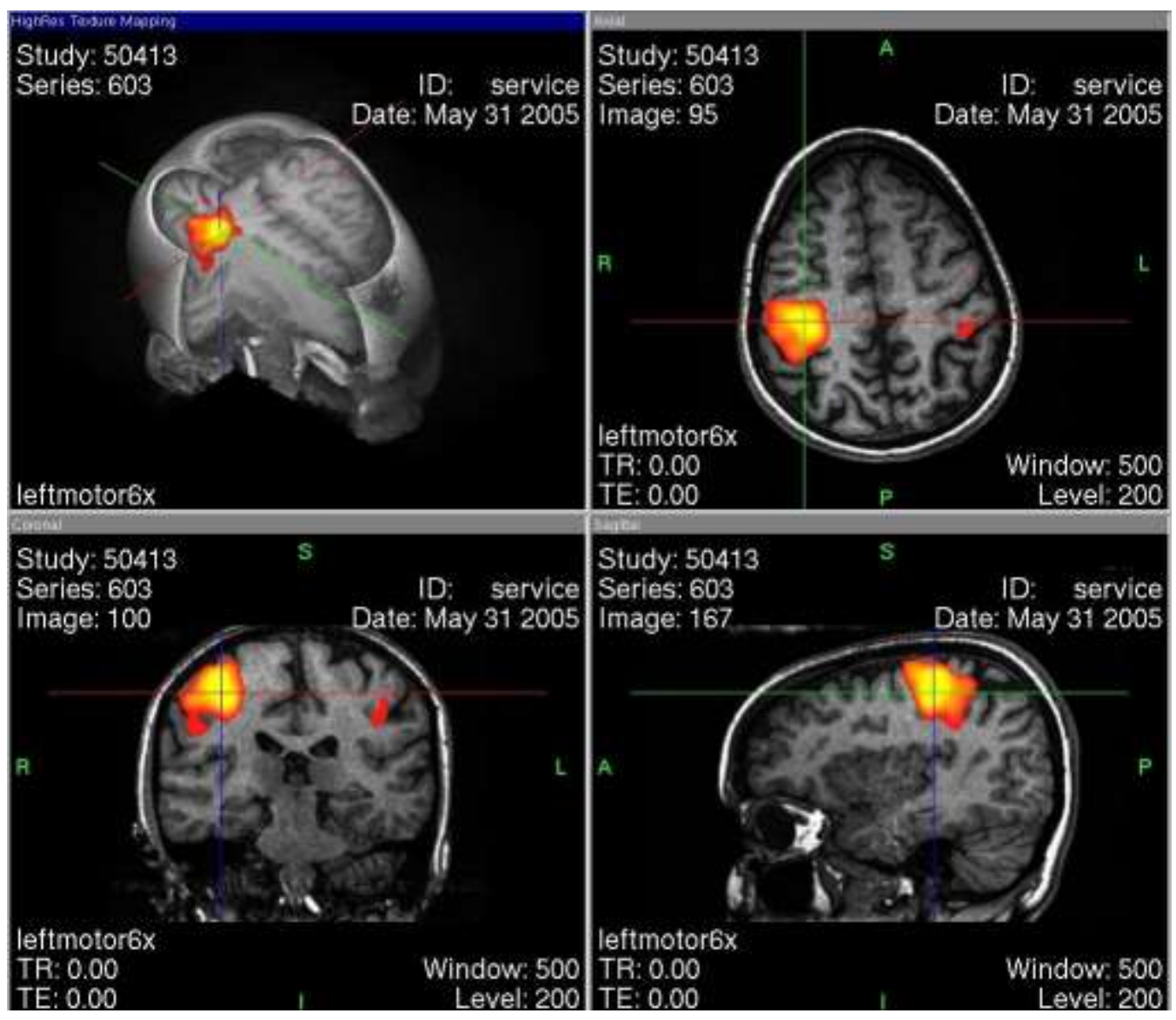

Figure 4. Overview of the Different Levels of Observation of a fMRI-scan after Left Finger Tapping. The Color-coded Area is an Indication of Increased Brain Activity

\subsection{Magnetic Resonance Angiography (MRA)}

Magnetic resonance angiography - also called a magnetic resonance angiogram or MRA. It is a modern imaging technique to detect strokes in the brain. If a neurologist believes that you may have a narrowing or blockage of blood vessels somewhere in your body, he or she may recommend a magnetic resonance angiography [18]. It is a group of 
vascular imaging techniques able of illustrate the extracranial and intracranial circulation. To identify any presence of blocking or narrowing of the arteries in the neck and brain. MRA is also used by neuroradiologists investigate carotid arteries in the neck and the cerebra vessels in the brain and can show shape, size, location and orientation.

To catch the picture of blood vessels MRA uses a powerful magnetic field and radio waves just like an MRI. In the condition of acute stroke, these imaging techniques are useful for validating stroke etiology and assessing vascular flow dynamics. MRA is typically partition into non-contrast and contrast-enhanced techniques. Non-contrast technique of MRA can be captured with phase contrast (PC) or TOF techniques and the two can be acquired as $2 \mathrm{D}$ slabs or 3D volumes that can help neurosurgeons to get very precise information about stroke.

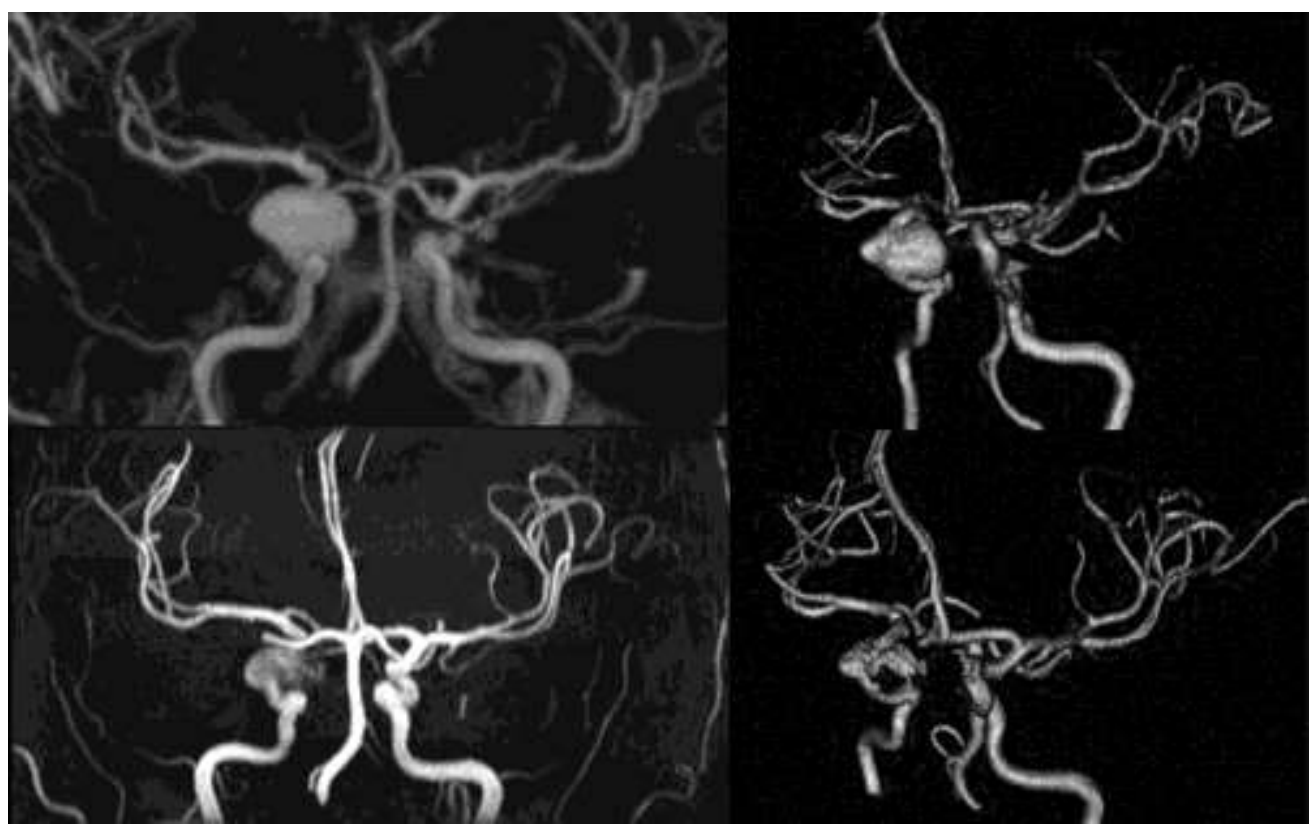

Figure 5. Coronal Oblique MIP and Volume-rendered Projections from Contrast-enhanced MRA (upper row) and TOF-MRA (lower row) Show a Large $\left(16^{\star} 12 \mathrm{~mm}\right)$ Aneurysm Arising from the Cavernous Portion of the Right Internal Carotid Artery

\section{Brain Surgery}

The opening step in treating the bengin tumor, malignant tumor and stroke is the surgery [21]. Brain surgery is an invasive procedure and closely associated with side effects. However, the side effects increases drastically when it comes to the surgery of the brain as it is the master of all body controls.

The severity and period of the side effects varies in accordance with the reason for the surgical procedure and the area of brain on which the surgical procedure was carried out. Bringing together the imaging technologies with tracking systems for surgical instruments will allow neurosurgeons to navigate the body. Over the past few decades the most influential advancement in the neurosurgery has been shift towards minimally invasive surgery (MIS) procedures. This revolution has been driven by the associated reduction in trauma to the patient as an outcome of smaller incisions, which translates in shorter hospital stays and reduces the recovery times. 


\subsection{Skull base Surgery and Open Surgery}

Skull base surgery and open surgery (craniotomy) are the traditional procedure to operate tumors (Bengin and Cancerous) and stroke. In these surgeries disc of bone is extract from the skull using specific tools to allow access to the underlying brain [22]. These are problematic procedure because it gives a high expose to the brain which leads to the high risk of infection and cause brain damage.

\subsection{Minimally Invasive Surgery}

Now a day's minimally invasive surgery is becoming more and more common in hospitals. The procedures performed in this surgery use small incision instead of one large opening. Because the incision is small, recovery is quick and less discomfort than with conventional surgery all with same benefits

To operate with less injury to the body than with open surgery, neurosurgeons use a variety of techniques in MIS. The finest of this surgery is that it is safer than open surgery and allows the patient to recover faster and heal with less pain and scarring [3, 4]. Precision, small entry point and short time keep safe the healthy tissue nearby and decrease pain after surgery and fast recovery time in comparison to the craniotomy.

MIS continues to be obstructed by several issues. In extension to hardware modernization, Computer Assisted Surgical surgery has been proposed as a way to overcome some of its current issues.

\subsection{Computer-assisted Surgery (CAS)}

(CAS) represents a surgical approach and a cluster of methods, that use computer technology for neuro-surgical planning, and for guiding or performing surgical interventions [23]. CAS is also known as computer-aided surgery, computer-assisted intervention, image-guided surgery and surgical navigation. When different types of computer-assisted surgical systems combined with minimally invasive surgery, it can be used for pre-operative planning, surgical navigation and to assist in performing surgical procedures.

\section{MIS Surgeries in India}

Minimally invasive surgeries are offering a ray of hope for the patients suffering with severe tumors and stroke problems. Minimally Invasive Surgeries are done by making two or three small openings and the patient can return to his normal routine in a short span [5,7]. In India the CyberKnife Robotic Radiosurgery, Gamma Knife radiosurgery, Endovascular neurosurgery, Interventional neuroradiology, Robotic-assisted surgeries are the some computer assisted minimal invasive surgery used to treat tumors and stroke.

\subsection{Neuroendovascular Surgery (Stroke)}

During neuroendovascular surgical procedures, an interventional neuroradiologist navigates a microcatheter (thin tube) through the vascular system. The catheter is inserted through a small incision in the groin, and then guided to the affected blood vessels for treatment [24].

Intracranial angioplasty and stenting are used to restore blood flow through blocked vessels inside of the skull. For angioplasty, the microcatheter is equipped with a tiny balloon on the tip. During the procedure, the catheter is navigated through the blood vessels to the occlusion, where the balloon is inflated to open the blockage. In some cases, a stent is placed to keep the blood vessel open.

In Carotid Angioplasty and Stenting, the carotid artery is a main artery on the neck, which delivers blood to the brain. Narrowing or blockage of the carotid artery causes more than 10 percent of acute ischemic strokes in the nation. Carotid angioplasty and 
stenting is a technique used to open a blocked carotid artery. In this procedure, interventional neuroradiologists insert a microcather through a small incision in the groin and up through the vascular system. Once it reaches the blockage, the balloon is inflated to open the vessel, and the stent is placed to keep it open.

\subsection{Interventional Neuroendovascular Surgery (Stroke)}

Within radiology Interventional neuroradiology is a subspecialty that also use catheters and radiology to diagnose and treat neurological conditions and diseases [25]. Intraarterial tPA: In the intra-arterial tPA procedure, the neurosurgons inject a thin, flexible catheter into an artery (femoral artery) and guides it up to the region of the clot then a administers the tPA through the catheter. Stroke patients can be treated for up to six hours when tPA is a administered intra-arterially at the site of the blockage because the blood flow would begin immediately.

\subsection{CyberKnife Robotic Surgery (Brain Tumor)}

The CyberKnife System is composed of a radiation delivery device, called a linear accelerator (or Linac), which is mounted on a robotic arm $[2,8]$. Robotic arm flexibility allow the CyberKnife System to pass radiation to tumors anywhere in the body, including the brain, head and neck, spine, lung, prostate, liver, pancreas, breast and other soft tissues [9]. Sophisticated software and advanced medical imaging are the key features of CyberKnife surgery which helps in tracking of tumor and patient movement. To ensure the treatment is delivered with a high degree of accuracy and precision high dose of radiations delivered in a regulated manner.

\subsection{Gamma Knife Surgery (Brain Tumor \& Stroke)}

Imaging studies, such as magnetic resonance imaging (MRI), computed tomography (CT), or angiography, are then obtained and the results are sent to the Gamma Knife's planning computer system. Team of physicians (radiation, oncologists and neurosurgeons) and medical physicists delineate targets and normal anatomical structures. A computer program is used for planning to determine the exact spatial relationship between the target, normal structures and the head frame to calculate Gamma Knife ${ }^{\circledR}$ treatment parameters [6]. Targets often are best treated during the treatment session with combinations of several successive highly focused treatments, commonly known as "shots". For an optimal plan and adequate dose determination, treatment parameters are adjusted by the physicians and physicists.

\section{Conclusion and Future Work}

No wonder a brain tumour and stroke is notoriously difficult to diagnose. Imaging technologies such as CT, MRI, MRA, fMRI detect the early stages of tumors and stroke in the body with detailed information. By utilizing imaging technologies, smart instruments and intuitive software, Minimal Invasive Surgery delivers greater surgical precision with minimum damage to brain. In future new technologies will introduce in MIS such as single-incision laparoscopy and natural orifice transluminal endoscopic surgery (NOTES), in which a tube is passed through a natural orifice to avoid making any incisions at all, promise to make procedures even gentler. On the cutting edge or, perhaps, the non-cutting edge -- the use of extremely minuscule surgical robots could make operations even more precise. 


\section{References}

[1] Shweta Suran,Vishwajeet Pattanaik, D.Malathi, "Discovering shortest path between points in cerebrovascular system", ACM , $6^{\text {th }}$ IBM Collaborative Academia Research Excahnge Conference(ICARE) 2014. DOI : 10.1145/2662117.2662122

[2] Vishwajeet Pattanaik,Shweta Suran, "Inducing human like motions in robots", ACM , 6 ${ }^{\text {th }}$ IBM Collaborative Academia Research Excahnge Conference(I-CARE) 2014. DOI : $10.1145 / 2662117.2662118$

[3] Tanio K. Tanev, "Minimally-invasive-surgery parallel robot with non-identical limbs", Mechatronic and Embedded Systems and Applications (MESA), 2014 IEEE/ASME 10th International Conference. DOI: 10.1109/MESA.2014.6935558

[4] B. Estebanez, G.Jimenez, V.Muýoz, "Minimally invasive surgery manoeuvre recognition based on surgeon model", Intelligent Robots and Systems, 2009. IROS 2009. IEEE/RSJ International Conference. DOI: $10.1109 /$ IROS.2009.5354157

[5] Jianmin Li, Xiaofei Wang, "Development of a novel mechanism for minimally invasive surgery", Robotics and Biomimetics (ROBIO), 2010 IEEE International Conference. DOI: $10.1109 /$ ROBIO.2010.5723529

[6] J.Dankelman, "Surgical robots and other training tools in minimally invasive surgery", Systems, Man and Cybernetics, 2004 IEEE International Conference. DOI: 10.1109/ICSMC.2004.1400699

[7] Alejandro Cuevas, Daniel Lorias, Arturo Minor, "Minimally invasive surgical skills evaluation in the field of otolaryngology", Computer-Based Medical Systems (CBMS), 2013 IEEE 26th International Symposium. DOI: 10.1109/CBMS.2013.6627769

[8] J.K. Hsu, Temei Li, S. Payandeh, "On integration of a novel minimally invasive surgery robotic system", Advanced Robotics, 2005. ICAR '05, Proceedings, 12th International Conference. DOI: 10.1109/ICAR.2005.1507447

[9] Ke Liang, Jianmin Li, Kang Kong, "Control system design of a novel minimally invasive surgery robot", Complex Medical Engineering (CME), 2011 IEEE/ICME International Conference. DOI: 10.1109/ICCME.2011.5876771

[10] M. Kaiser, J. Krug, G. H. Rose, "Interventional MRI: Minimal-invasive Surgery under MR guidance", Microwave Symposium Digest (MTT), 2011 IEEE MTT-S International. DOI: 10.1109/MWSYM.2011.5972988

[11] A.R. Cysewska - Sobusiak, A. Sowier, P. Skrzywanek, "Application of combined methods of imaging in minimally invasive surgery", Engineering in Medicine and Biology Society, 2003. Proceedings of the 25th Annual International Conference of the IEEE. DOI: 10.1109/IEMBS.2003.1279423

[12] Sohrab Eslami, Gregory S. Fischer, Sang-Eun Song, "Towards clinically optimized MRI-guided surgical manipulator for minimally invasive prostate percutaneous interventions: constructive design", Robotics and Automation (ICRA), 2013 IEEE International Conference . DOI: 10.1109/ICRA.2013.6630728

[13] Yacine Kabir, A. Belhadj-aissa, "Analysis of stroke Magnetic resonance images", Information and Communication Technologies: From Theory to Applications, 2008. ICTTA 2008. 3rd International Conference. DOI: 10.1109/ICTTA.2008.4530104

[14] Na Geng, Xiaolan Xie, "Simulation study of Magnetic Resonance Imaging examination reservation processes for stroke patients", Control Conference (CCC), 2012 31st Chinese. NSPEC Accession Number: 13221670

[15] Hayder Saad Abdulbaqi, Mohd Zubir Mat, Ahmad Fairuz Omar, "Detecting brain tumor in Magnetic Resonance Images using Hidden Markov Random Fields and Threshold techniques", Research and Development (SCOReD), 2014 IEEE Student Conference. DOI: 10.1109/SCORED.2014.7072963

[16] Shobana G, Ranjith Balakrishnan, "Brain tumor diagnosis from MRI feature analysis - A comparative study", Innovations in Information, Embedded and Communication Systems (ICIIECS), 2015 International Conference. DOI: 10.1109/ICIIECS.2015.7193137

[17] Sérgio Pereira, Adriano Pinto, Victor Alves, "Brain Tumor Segmentation Using Convolutional Neural Networks in MRI Images", IEEE Transactions on Medical Imaging. DOI: 10.1109/TMI.2016.2538465

[18] Jeyaraj Durai and Paulin Sudhan, "Stroke Epidemiology and Stroke Care Services in India", Journal Of Stroke. DOI: 10.5853/jos.2013.15.3.128.

[19] Centers for Disease Control and Prevention, National Center for Health Statistics. Underlying Cause of Death 1999-2013 on CDC WONDER Online Database, released 2015. Data are from the Multiple Cause of Death Files, 1999-2013, as compiled from data provided by the 57 vital statistics jurisdictions through the Vital Statistics Cooperative Program: http://wonder.cdc.gov/ucd-icd10.html. Accessed on Feb 3, 2015. 2.

[20] Mozzafarian D, Benjamin EJ, Go AS, et al. on behalf of the American Heart Association Statistics Committee and Stroke Statistics Subcommittee. Heart disease and stroke statistics-2016 update: a report from the American Heart Association. Circulation. 2016;133:e38-e360.

[21] A.Boyer, J.Deverdun, H.Duffau, et al, "Crossed cerebellar diaschisis after awake brain surgery: Can we measure pre/post operative changes on resting state fMRI data?" , Neural Engineering (NER), 2015 IEEE . DOI: 10.1109/NER.2015.7146803. 
[22] Sungmin Kim, Hyun Jae Kang, et al, "Photoacoustic image guidance for robot-assisted skull base surgery", Robotics and Automation 2015 IEEE. DOI: 10.1109/ICRA.2015.7139239.

[23] Xi Wen, Hong Wan ,et al, "Intelligent Coordinate Registration Method for Computer-Assisted Surgery", Computational Intelligence and Communication Networks (CICN), 2015 IEEE. DOI: 10.1109/CICN.2015.129.

[24] Irene Cheng, Rui Shen, et al, "An augmented reality framework for optimization of computer assisted navigation in endovascular surgery", Engineering in Medicine and Biology Society (EMBC), 2014 IEEE. DOI: 10.1109/EMBC.2014.6944908.

[25] Shuxiang Guo, Yuan Wang, et al, "An improved master-slave interventional surgery robotic system with force feedback", Mechatronics and Automation (ICMA), 2016 IEEE. DOI: 10.1109/ICMA.2016.7558599 
International Journal of Bio-Science and Bio-Technology Vol.9, No.3 (2017) 